The consideration of these questions or of the others which I avoided in the first words of this paper, while important, would now only befog what I have sought to make the sole question here. It is this: should craniotomy ever be done in those cases in which there can be and should be a deliberate election between it and a Cæsarian section? I vote-No.

One thing is most vividly brought to my thought by the consideration of this matter. It is the necessity for the more thorough training of medical students that they may recognize early when induced labor is likely to be required and to be successful, and when it is better to do a Cæsarian section and how to do it. They are taught to induce labor, they are taught how to do craniotnmy; they are not always well taught as to how to do Cæsarian section and the Porro operation, and they are not always well taught as to when induced labor is likely to be successful, or how to recognize the cases in which they must be prepared to do, or have done, a Cæsarian section, either just before or at the onset of labor. I would not advocate that every man who is likely to attend a labor case should attempt to do Cæsarian section, neither would I advocate, did I believe craniotomy justifiable, that every man should attempt to do craniotomy. But I do believe that no man should be permitted to graduate from our medical schools, who has not such a knowledge of pelvimetry and what it means but that he shall know just what can be expected from and what must be done with every case under his care and know enough, even should he not feel competent to attempt an obstetrical operation himself, to have the services of someone for his patient who is able, not only to save her life, but also to deliver her of a living child.

In these days when every small city has its gynæcologist, fit and able and willing to do Cæsarian section, either at home or away from home, either for a fee or without it; when every little city has its lospital, public or private, where such an operation can be done if the patient's home is to humble for the necessities for it, there can be, in my judgment, but very rarely excuse for the sacrifice of human life made necessary by failure to recognize before hand the conditions necessary to be met and to use the means required to overcome them.

\section{THE TREATMENT OF POSTERIOR ROTATION OF THE OCCIPUT DURING LABOR.}

Read in the Section of Obstetrics and Diseases of Women, at the Fortythird Anuual Meeting of the American Medical Association, held at

BY EDWARD P. DAVIS, A.M., M.D.,

Professor of Obstetrics and Diseases of Infancy in the Philadelphia Polyclinic; Clinical Lecturer on Obstetrics in Jefferson Medical College; Obstetrician to the Philadelphia Hospital, ete.

Among the complications of labor not requiring surgical interference, none is more important or fraught with greater danger to child and mother than posterior rotation of the occiput. We desire to substitute this term for that commonly employed - "occipito-posterior positions"-believing that the method of obstetric study that recognizes but two positions for each presentation is more rational and serves a better purpose than the more complicated system that calls for many positions. We shall then make but two positions for each presentation. For example, a vertex presentation has a first and a second position. The first is the usual one, the back of the child directed toward the left side of the mother, and the vertex directed slightly toward the left side of the mother's pelvis. The second position of a vertex presentation is that in which the back of the child is directed toward the mother's right side, and the occiput points to the right of the mother's pelvis.

During labor the occiput, in the vast majority of cases, turns forward, toward the symphysis pubis. Occasionally it descends through the pelvis, showing a tendency to turn posteriorly, thus giving rise to what have been styled "posterior positions." It occasionally happens that during pregnancy the occiput is situated behind the centre of the mother's pelvis; whether the occiput endeavors to turn backward, or whether during pregnancy it is situated slightly behind the centre of the pelvis, it is more rational and easier of comprehension to call this abnormal turning of the occiput "posterior rotation" than to make four positions, two of them posterior. In common with many Continental students of obstetrics, we style the condition as an "abnormal rotation of the occiput," and not as "occipito-posterior positions."

The causes of the tendency to turn posteriorly are several. The mother's pelvis may be narrowed in its anterior half and its sides may lack those surfaces anteriorly to a line drawn upward and forward from the spines of the ischia that normally favor the anterior turning of the head. The head of the child may be of unusual size or contour, finding its most convenient lodgment at the posterior extremity of one of the oblique diameters of the pelvic brim. Again, during labor, the posture assumed by the mother may not be such as to favor the anterior turning of the vertex. Many cases exist in which no one factor has been found to be entirely responsible for the abnormality; thus, a small child may enter the pelvis with its head in partial extension or flexion, and this intermediate condition remaining, the occiput may turn toward the hollow of the sacrum.

Some of the symptoms of posterior rotation of the occiput may be perceived before labor has actually begun, while others are discernible only after the commencement of labor. Palpation and auscultation will often reveal the fact that the occiput is directed toward the middle line or even slightly behind the centre of the pelvic brim. The back of the child may be turned somewhat posteriorly, so that the heart-sounds seem to come from deep in one side of the mother's abdomen. If the abdominal walls are thin, the occiput can be mapped out and accurately located. If a history of previous labors be sought, there will be an account of prolonged parturition, probably ended by instrumental delivery, and often resulting in the death of the fœtus.

At the time of labor it is often difficult to map out accurately the position of the head unless the tissues be elastic and admit of manipulation. If the attending physician notices the absence of the vertex in the anterior half of the mother's pelvis, he will do well to allow her to inhale sufficient chloroform to permit the introduction into the.vagina of enough of the hand to make a positive diagnosis.

We shall best understand the indications for treatment by considering briefly the course of a hypothetic case if left to nature. Statistics show that the greater number of cases result in anterior rotation of the occiput without assistance. By some the proportion of cases that rotate spontaneously to the front is placed as high as 96 per cent.; others allow but 75 
per cent. My own observation and the records of 219 cases occurring at the Philadelphia Hospital, lead me to believe that 87 per cent. of these cases th terminate spontaneously with anterior rotation. A source of fallacy in a study of the question arises from the fact that an exact diagnosis of the position of the head is rarely made early in labor; a practitioner is often satisfied with finding that the head is presenting and that the face is not the part found by the examining finger. Thus, a case in which posterior rotation has begun, but which terminates by turning forward, is not recognized until anterior rotation has occurred.

If we seek to ascertain the factors upon which anterior rotation depends, we find them to be essentially three in number: First, a normal proportion in size between the head and the pelvis; thus, a small head in a small pelvis favors normal rotation, and a large head in a large pelvis is likewise favorable. Secondly, a flexed position of the head is essential to anterior rotation of the occiput. Thirdly, the forces of labor must be normal, namely: the contractions of the uterus and the resistance afforded by the pelvic floor. If any one of these factors is notably deficient, a perversion of rotation is very likely to occur.

Abnormal rotation of the occiput is a source of danger to the life of the child and to the integrity of the mother's tissues, and also to her life. The child's life is jeopardized by long-continued pressure during prolonged labor, resulting in visceral hæmorrhage and death. The mother's tissues are endangered by reason of the abnormal prolongation of the pressure of the head during efforts at rotation, and her life is imperiled by the added risk of septic infection and exhaustion.

The treatment of such abnormal rotation would rationally consist in an effort to supply the one or more factors already described that may be deficient or perverted. Although the physician cannot remedy disproportion in size between the head and the pelvis, the sooner he becomes aware of such disproportion the better for his patient. No woman should be attended during her first pregnancy unless her pelvis has been measured by her medical attendant. In making this statement we do not insist upon an elaborate measurement of the pelvis, but urge that three external measurements be invariably made. These are: the external conjugate, the distance between the anterior superior iliac spines, and the distance between the outermost points of the iliac crests. If the physician has the opportunity of making such an examination as early as the thirtieth week of gestation, he may, if necessary, be able to induce labor, thus securing a normal rotation of the occiput before disproportion between the head and the pelvis has gone so far as to endanger his patient. If he does not see his patient until she is considerably advanced in labor, the knowledge that her pelvis is smaller than the average will be of great value in preventing the futile effort to deliver a large head through a pelvis too small for it. In addition to the measurement of the mother's pelvis, an effort should be made to ascertain the comparative sizes of the head and the pelvis. This can be done by placing the patient upon her back, her thighs being flexed, while the fotal head is gently pressed downward into the pelvis by the examining hand laid broadly behind the pubes, the other hand steadying the fundus of the uterus. If more exact information is desired, one hand may be placed against the head by internal examination, while pressure is exerted with the other hand behind the pubes. A head that begins to slip into the brim of the pelvis under such manipulation is not too large to rotate successfully during labor.

Flexion of the head, necessary to secure anterior rotation, may be promoted and maintained by the employment of the hand, accompanied by the use of forceps. When the physician discovers that the occiput is turning posteriorly, while the head is but slightly flexed, if the patient be anæsthetized sufficiently to permit the introduction of the hand, the occiput may then be drawn down, or the chin pushed upward, and flexion secured. If this effort fails, flexion may be secured by axis-traction with the forceps, as will be described in a succeeding paragraph.

Flexion and anterior rotation may be further facilitated by the posture of the patient; thus, if the back of the child be turned toward the left side of the mother and the occiput tends to rotate posterior$1 y$, the mother should be turned upon her left side, with her thighs flexed and her shoulders bent slightly forward. This posture causes the fundus of the uterus, containing the body of the foetus, to fall toward the left side of the mother's spinal column, as it will be remembered that the uterus normally occupies a position of right lateral obliquity in the abdomen of the mother. This movement of the fundus from right to left, by gravitation, favors the turning of the occiput from left to right, and thus anterior rotation of the vertex is expedited. If the child's back is directed toward the right side of the mother and the occiput seems about turning toward the sacrum, the mother should be placed upon her right side, with her body fiexed as has been described and her right side propped up from the bed by means of pillows. Gravitation of the fundus is thus favored, and anterior rotation is more likely to occur.

The posture often instinctively assumed by women, namely, that of kneeling strongly forward upon the bed, is one that favors flexion and anterior rotation. The fundus of the uterus falls forward, the head tends to flex and descend into the pelvic cavity, and the long axis of the foetus corresponds more perfectly with the direction of the axis of the pelvis.

In addition to posture, constant external pressure against the vertex sometimes favors anterior rotation of the occiput. Thus, I remember having seen a case so treated in the wards of a foreign hospital; the patient was placed upon her side and a small bag filled with sand was laid upon the abdomen against the vertex.

We now come to consider the last of the three conditions upon which anterior rotation depends, namely, the expulsive force of the uterus and abdominal muscles, and the resistance of the pelvic floor. If the former is deficient, a suitable stimulant to the nervous system is indicated: thus tea, coffee, alcohol and quinine are most available; it is rarely necessary to employ ergot, and exceptionally the faradic current of electricity has been found useful. A narcotic that causes labor to cease absolutely for a time, while the patient sleeps, has often been found of value, the patient on awakening rousing to renewed activity. Direct stimulation of the uterus by rubbing the abdomen, and by pressure exerted upon the head in the axis of the pelvis, may carry to a successful parturition a labor lingering in its last stage. Again, anæsthesia of a moderate degree may temporarily re- 
move the depressing influence of pain and cause renewed uterine activity.

necessary. As traction is made, the tapes tend to

While such expedients are frequently successful, yet the forceps is very often the last resource of the practitioner. In this, as in all forceps deliveries, two methods are to be distinguished. One is that which all that use forceps desire to carry out, namely, the application of the blades accurately to the sides of the child's head; this requires a forceps modelled after French instruments, or shaped like the familiar blade devised by Davis, of London.

The other method of applying the forceps is that recognized as a valuable expedient, although not the ideal method of operating; this is the application of the forceps to the sides of the pelvis, and suggests the employment of an instrument modelled after the forceps of Simpson, of Edinburgh. In the first instance, forceps and head rotate together; in the second, the head rotates in the forceps, the blades remaining in the axis of the pelvis at the sides of its cavity. The first method requires for its safe performance a very accurate diagnosis of the precise location of the occiput; this will usually require anæsthetizing the patient and the introduction of nearly all or of the entire hand within the vagina. In the second method the occiput may not be definitely located, although its absence from its usual location may have been determined. The forceps is then applied to the sides of the pelvis, traction is made gently with the pains, and between the tractions the grasp of the forceps is relaxed, the head rotating within the forceps-blades, so that each application is virtually a new one.

Neither use of the forceps is skilful that does not at the same time employ axis-traction. All obstetricians are familiar with the simple manipulation of performing axis-traction with the hands, grasping the handles of the forceps with one hand and raising them, while the other presses downward and backward upon the instrument at the shank. If the case be a difficult one, this requires for its successful performance very considerable strength in the hands and wrists. The axis-traction forceps of Tarnier and those modelled after it represent an established method of securing axis-traction in these cases. The Tarnier instrument, however, is not convenient for the ordinary exigencies of confinement; it is too heavy, too large, and too expensive. Poullet, of Lyons, in a thesis described the application of the tapes as tractors to secure axis-traction. Many had already employed this, or a similar device, by passing a tape or cord around the shank of the forceps, and forming a loop into which the foot of the operator could rest, and by downward traction assist in the delivery. Poullet has modified and made more simple his use of the tapes, as shown in his most recent instruments. I applied the tapes, as first suggested by Poullet, to the Simpson forceps for the purpose of meeting the indications in those cases in which the occiput tends to rotate posteriorly, and in which the Simpson forceps applied to the sides of the pelvis seems most clearly indicated. The tapes have been similarly attached to the Davis forceps for cases in which that instrument seems most suitable; my experience leads me to prefer the Tarnier forceps in cases in which the instrument can be applied accurately to the sides of the head.

In using the forceps with the tapes no extra device for holding the instrument tightly upon the head is draw the blades together; thus, in using the Simpson forceps, care should be exercised that the blades do not approach too closely.

In using either form of instrument, but moderate force is needed to effect delivery, provided axis-traction be made; the failure to perform axis-traction accounts for many of the difficult and dangerous cases of forceps delivery. The head should be kept thoroughly flexed during its exit from the genital tract; the use of axis-traction has largely done away with that function of the forceps said to favor rotation. No one would attempt to forcibly twist a vertex in the pelvis; the difference between traction in the axis of the pelvis and the attempt to deliver without such traction can never be appreciated without a practical experience with the two methods. The former is a difficult, tedious, and laborious task; the latter is a matter of much less difficulty. As illustrating the method of favoring rotation by axistraction with the Simpson forceps the following case, reported originally in the first volume of the Philadelphia Hospital Reports, may be described: ${ }^{1}$

M. H., thirty-six years, an Irish domestic, unmarried, a unipara, was admitted to the hospital October 16,1889 , in a fairly good condition. While awaiting delivery, she suffered from numerous severe attacks of asthma, and was found to have emphysema, with some dilatation of the right ventricle. Examination of the heart revealed no valvular lesion, and examination of the urine showed an absence of albumin and of casts. For the relief of the asthma, grindelia robusta, potassium nitrite, and soda, with chloroform-inhalations, were employed. During the woman's stay in the obstetric ward her general condition did not improve, the attacks of asthma increasing in frequency, the patient becoming anæmic, irritable, and weak. About three weeks before labor, albumin in considerable amount was found in the urine, and about a week later the legs and feet became odematous.

Labor began Tuesday, December 24, at 10:30 P.M., the patient reaching the Maternity ward at $10: 30$ P.M., December 25, 1889: At this time she was weak and irritable; she suffered from dyspepsia, and complained of pain in the back. The os was patulous to one finger; no presenting part could be felt, polyhydramnios being present; the uterine contractions were infrequent, of slight force, but caused great suffering. The first stage of labor lasted sixty-two hours, the general condition of things remaining much as described; the abdomen was enormously distended, tense, fluctuating; the fetal heart was distinctly heard low down in the right flank. The position of the fetus could not be made out by palpation. The patient became weaker, the pulse losing in volume and increasing in frequency to 115. At an early hour in the morning of December 28 , the membranes ruptured, and a large amount of greenish liquor amnii escaped, but the head, which presented in the right occipito posterior position, prevented the escape of all. It was now found that the woman's bladder was full of urine, and catheterization was necessary. Still the head failed to descend through the pelvis, which was not markedly contracted, the measurements being: Spines, $23.5 \mathrm{~cm}$.; iliac crests, $26.5 \mathrm{~cm}$.; external conjugate, $18 \mathrm{~cm}$; right diagonal, $22 \mathrm{~cm}$.; left diagonal, $21 \mathrm{~cm}$; ; trochanters $28.5 \mathrm{~cm}$; ; circumference, 85 $\mathrm{cm}$.

The contractions of the uterus diminished in force, and at $12: 30$ P.M., December 28, the patient was anæsthetized with chloroform; Simpson's forceps, with the Poullet tapes attached, was applied, and the head delivered. The cord was wound twice around the neck. Delivery was difficult, consuming considerably over an hour; occiput remained posterior until just before delivery, when it quickly rotated forward. The grasp of the forceps was relaxed between the tractions to allow rotation. Double episiotomy was practiced and no considerable laceration occurred.

In bringing this paper to a conclusion, I desire first to draw attention to some errors that I think not uncommon in the treatment of these cases. Of primal importance in this report is the neglect I Philadelphia Hospital Reports, vol. i, p. 229. 
of the effort to measure the patient's pelvis of usage on the Continent of Europe, and which will when possible, and when this is not practicable to render more comprehensible the mechanism of labor. estimate the comparative sizes of the head of the I refer to the designation of two positions only for child and the mother's birth-canal. In this the pro- each presentation, anterior or posterior rotation befession must educate its patients, and this can be ing designated, as the case may be. Second, the imdone only if methods of examination are conducted portance of pelvimetry and of the comparative estiwithout exposure, without violence, and in a kindly mation of the sizes of the head and the pelvis is and skilful manner. It is my custom to teach students to examine the pelvis, diagnosticate the position of the child and its presentation, and ascertain whether the head is likely to enter the pelvis readily, while the patient remains covered with one thickness of linen. We can no longer comfort ourselves with the remark that American-born women rarely have contracted pelves; this statement is not strictly true, and the large number of foreigners coming to our country show frequent examples of varying degrees of pelvic malformation. The skilful obstetrician, by his knowledge, avoids prolonged and disastrous labors, and wuch good results are impossible without a skilful examination of the pregnant patient. If a large head be detected at the brim of the pelvis, scarcely able to enter, and showing a disposition to rotate posteriorly, it will be found better practice in many cases to perform the conservative Crsarean section, saving the lives of mother and child, rather than subjecting the child's life to great risks and exposing the mother to serious injury in delivery.

I desire, secondly, to draw attention to the efficacy of posture in favoring rotation of the occiput when the head has entered the pelvis. Whether it be that the patient lies upon the side toward which the occiput is pointing, or whether she inclines the pelvis forwards, I am convinced from observation that posture is a valuable adjunct in the conduct of such cases. I desire also to criticise that method of examination consisting in the introduction of but a portion of one or two fingers in the effort to make an accurate diagnosis of the location of the occiput, and to favor its anterior turning. The practitioner should prepare himself to apply the forceps if necessary, and then anæsthetize his patient to obstetric ancesthesia, preferable with chloroform; he should introduce so much of the hand as may be necessary to make an accurate examination of the entire vertex. In the intervals between the pains he will be able to favor anterior rotation, and when he has thus brought the vertex in front of the middle of the pelvis, without removing the hand the forceps may be applied and the labor terminated.

Third. The forceps is often applied too soon, thus neglecting one important factor in anterior rotation, namely, the resistance of the pelvic floor. If the forceps be applied before the head has had an opportunity to rotate upon the pelvic floor, its posterior turning is often favored by the instrument rather than its anterior rotation. On the other hand, the forceps is often applied too late, when flexion has become impossible by reason of the persistence of partial extension, the head becoming impacted upon the pelvic floor in this unfavorable position. The life of the child has frequently been lost in such cases before the final effort at delivery is made, and when such is the case craniotomy is often the only rational procedure.

In conclusion, I would urge the following as points of importance: First, the adoption of the simple nomenclature already stated, which has borne the test paramount; by the second of these I mean the careful estimation as to the degree of engagement of the head, and if the head has not engaged, pressing it gently into the pelvis by supra-pubic pressure, the patient being, if necessary, partially anæsthetized. Third, when the head is proportionate in size to the pelvis, and lingers upon the pelvic floor by reason of failure in expulsive force, the child's life and also the mother's safety being in danger, the forceps should invariably be used with axis-traction. The forceps should be used in accordance with the possibilities of the case, and that form of instrument should be selected that was intended for such possibilities. Whenever practicable, forceps designed to be so applied should be accurately placed upon the sides of the child's head, axis-traction made, the head and the forceps rotating together anteriorly. When such accurate apposition is impossible, forceps modelled for application to the sides of the pelvis should be employed with axis-traction, the forceps making as little pressure as possible upon the head, the blades being relaxed after each traction so that rotation anteriorly may occur between the blades. Fourth, a conservative Cæsarean section may be indicated if a living child, with large, firmly ossified head, presents with a posterior rotation of the occiput, the head failing to descend into the pelvic cavity; craniotomy may be indicated if such a child has perished during its entrance in to the pelvis.

\section{THE IMPORTANCE OF SURGICAL TREAT- MEN'T FOR LACERATION OF THE CERVIX UTERI.}

Read in the Section of Obstetries and Diseases of Women, at the Fortythird Annual Meeting of the American Medical Association, held in Detroit, Mich., Juue, 1892.

BY AUGUSTUS P. CLARKE, A.M., M.D., OF CAMBRIDGE, MASS.

The opportunities now afforded to the gynecologist for studying the effect in any case of laceration of the cervix uteri leave but little doubt that treatment is a most important subject for consideration. Experience has, however, demonstrated that the local application of the various agents which from time to time have been suggested as being remedial or beneficial is at best but a temporary expedient. Whenever a laceration occurring at the cervix extends through the internal and external muscular tissue, the mucous coat lining the canal suffers materially from the violence. The plicæ palmatæ which have been described under the term arbor-vitæ undergo serious disturbance in their relation to the other tissues with which they are connected. This condition often leads to congestion, thickening and to induration of the parts involved and to more or less hypertrophy and malnutrition of the higher uterine segments, and to changed relation and to displacement of the lower cervical zone. When laceration occurs at the cervix it is not only that the muscular and the mucous structures are injured but it is also that the mucous glands which so freely abound in 